\title{
Adipositaschirurgie im Kindes- und Jugendalter: Wo stehen wir im Jahr 2008?
}

\author{
Holger Till $^{a}$ Susann Blüher ${ }^{b}$ Wieland Kiess $^{b}$ \\ ${ }^{a}$ Klinik und Poliklinik für Kinderchirurgie, Universität Leipzig, \\ ${ }^{\mathrm{b}}$ Klinik und Poliklinik für Kinder und Jugendliche, Universität Leipzig, Deutschland
}

\author{
Schlüsselwörter \\ Adipositaschirurgie · Jugendalter · Metabolisches Syndrom · \\ Leitlinien · Beobachtungsstudie
}

\section{Zusammenfassung}

Die Adipositaschirurgie bei Kindern und Jugendlichen findet in Deutschland bisher keine breite Akzeptanz, obwohl es auch bei uns zahlreiche Patienten gibt, die ihr Gewicht trotz intensiver Therapien nicht dauerhaft reduzieren können. Wie auch bei Erwachsenen weisen viele dieser Kinder und Jugendlichen bereits eine oder mehrere metabolische und/oder kardiovaskuläre Veränderungen des metabolischen Syndroms auf. Der folgende Artikel vergleicht die nationalen mit den internationalen Erfahrungen hinsichtlich der Leitlinien, Operationstechniken und Ergebnisse. Dabei zeigt sich, dass es in Deutschland bisher weder eine einheitliche Leitlinie noch ein zentrales Register für Adipositaschirurgie bei Kindern und Jugendlichen gibt. International gelten die Empfehlungen der Bariatric Scientific Collaborative Group (BSCG) auch für Kinder als Standard. Operativ finden sich in der Literatur im Wesentlichen der Roux-Y-Magenbypass und das Magenband, in letzter Zeit auch die Schlauchmagenbildung (sleeve gastrectomy). Diese werden insbesondere in den USA in designierten pädiatrischen Zentren durchgeführt. Deren Erfolgs- und Komplikationsraten scheinen ähnlich wie bei Erwachsenen zu sein. Allerdings bleibt dabei völlig offen, wie diese Ergebnisse angesichts der sehr viel längeren Lebenserwartung zu beurteilen sein werden. Als Konsequenz ergibt sich, dass für Kinder und Jugendliche mit morbider Adipositas a) spezielle Zentren mit dem gesamten Behandlungsspektrum etabliert werden sollten, b) Leitlinien für diese Adipositaschirurgie definiert werden müssen und c) eine langfristige Nachsorge aller Patienten im Rahmen einer zentralen Studie gewährleistet sein müsste, um Erfahrungen für die Zukunft zu generieren.

\section{Key Words}

Bariatric surgery · Adolescence · Metabolic syndrome · Guidelines · Observation study

\section{Summary}

Bariatric Surgery for Morbid Obesity in Childhood and

Adolescence: Where Do We Stand in 2008?

Bariatric surgery for children and adolescents with morbid obesity has not gained broad acceptance in Germany yet. Nevertheless, these children often fail to reduce weight despite intensive weight loss programmes and suffer from an associate metabolic syndrome, just like adults. Thus, bariatric surgery may be a favourable option. The present article compares national and international experiences concerning guidelines, surgical procedures, and results. It becomes obvious that Germany has neither specific guidelines for children and adolescents nor a central registry. Internationally, the recommendation from the Bariatric Scientific Collaborative Group (BSCG) should be taken as the standard. As in adults, most surgeons perform Roux-Y gastric bypass or gastric banding. Additionally, sleeve gastrectomy is gaining some popularity. These procedures are performed in designated pediatric centres especially in the US. Their success and complication rates are similar to those found in adults. The overall long-term consequences, however, remain unclear. Thus, for bariatric surgery in children and adolescents it may be concluded that a) these patients should be treated in designated centres that offer the full range of therapeutic options, b) specific guidelines should be established, especially in Germany, and c) a long-term postoperative study of all patients is necessary to collect all data and refine the techniques used.

$\begin{array}{ll}\text { KARGER } & \text { ( 2009 S. Karger GmbH, Freiburg } \\ \text { Fax +497614520714 } & \text { Accessible online at: } \\ \begin{array}{l}\text { E-mail Information@Karger.de } \\ \text { www.karger.com }\end{array} & \text { www.karger.com/ofa }\end{array}$

Prof. Dr. Holger Till

Klinik und Poliklinik für Kinderchirurgie

Frauen und Kinderzentrum der Universität Leipzig

Liebigstraße 20a, 04103 Leipzig, Germany

Tel. +49 341 97-2 64 00, Fax: -2 6409

holger.till@uniklinik-leipzig.de 


\section{Einleitung}

Die morbide Adipositas nimmt auch in Deutschland bei Kindern und Jugendlichen dramatisch zu [1-3]. Dabei leiden auch diese Patienten bereits an metabolischen Begleiterkrankungen wie Insulinresistenz und Diabetes Typ 2 (!), arterieller Hypertonie, Lipidstoffwechselstörungen, Hyperurikämie sowie orthopädischen oder psychischen Erkrankungen [4-7].

Ähnlich den profunden Erfahrungen bei Erwachsenen führen die meisten konservativen Therapieansätze (Ernährungsanalyse und -schulung, Steigerung der körperlichen Aktivität, psychologische und familientherapeutische Intervention, interdisziplinäre Kurmaßnahmen) oft nicht zum gewünschten längerfristigen Erfolg. Andererseits zeigen die Erfahrungen zur Adipositaschirurgie bei Erwachsenen, dass diese postoperativ nicht nur signifikant an Gewicht verlieren, sondern den niedrigeren BMI über 10-15 Jahre relativ stabil halten können [8]. Ferner bessern sich die Begleiterkrankungen signifikant, so dass die Gesamtmortalität bei extremer Adipositas im Vergleich zur konservativen Behandlung um $30-40 \%$ gesenkt wird $[9,10]$.

Für Kinder und Jugendliche liegen derzeit besonders in Deutschland vergleichsweise wenige Daten zur Adipositaschirurgie vor. Der folgende Artikel vergleicht die nationalen mit den internationalen Erfahrungen.

\section{Material und Methoden}

Die internationale Literatur (Datenbank PubMed, Internet) wurde hinsichtlich Indikationsstellungen, Techniken und Ergebnissen analysiert.

\section{Ergebnisse}

\section{Indikationen}

In Deutschland fehlen derzeit (noch) spezifische Leitlinien zur Adipositaschirurgie bei Kindern und Jugendlichen. Deswegen sollten die Richtlinien der Bariatric Scientific Collaborative Group (BSCG) $[11,12]$ als Maßstab gelten. Darin sind folgende Eckpunkte definiert:

Einschlusskriterien:

- BMI $>45 \mathrm{~kg} / \mathrm{m}^{2}$ bzw. 3,5 BMI-SDS oder BMI $>40 \mathrm{~kg} / \mathrm{m}^{2}$ und mindestens eine schwerwiegende bzw. zwei weniger schwerwiegende Komorbiditäten (Tab. 1)

- Keine Gewichtsreduktion durch ein 6- bis 12-monatiges ambulantes interdisziplinäres Therapieprogramm erzielt (Betreuung in einer Adipositassprechstunde, Kurmaßnahme, Sport, Ernährungsanalyse und -schulung, psychologische Betreuung)

- Indikationsstellung zur Operation durch interdisziplinäres Expertenteam (unter anderem Kinder- und Jugendarzt, Kinderpsychiater, klinisches Ethikkomitee)

- Bereitschaft und ausreichende Compliance zur erforderlichen Nachsorge, zu Substitutionsmaßnahmen und zu einer postoperativen Weiterbetreuung an einem Adipositaszentrum (für Kinder und Jugendliche).
Tab. 1. Häufige Begleiterkrankungen der Adipositas ${ }^{a}$

Schwerwiegende Komorbiditäten

Diabetes Typ 2 bzw. massiver Hyperinsulinismus und gestörte

Glukosetoleranz (IGT)

Obstruktive Schlafkrankheit

Arterielle Hypertonie

Weniger schwerwiegende Komorbiditäten

Arthrosen

Varikosis

Dislipidämie

Hyperurikämie

Gastroösophagealer Reflux

Bewegungseinschränkung

Pannikulitis

Steatohepatisis

${ }^{\mathrm{a}}$ Adaptiert aus [21].

Ausschlusskriterien:

- Schwerste psychiatrische Störungen (instabile Psychosen, Borderline-Persönlichkeit, schwere Depression und Persönlichkeitsstörungen)

- Diagnostizierte Essstörung

- Alkohol- und Drogenabusus

- Unfähigkeit des Patienten zur Teilnahme an einer langfristigen interdisziplinären Nachsorge.

Nach Erfassung der umfangreichen medizinischen, psychosozialen und ernährungsassoziierten Befunde sollte eine interdisziplinäre Fallbesprechung erfolgen. Insbesondere bei jungen oder mental retardierten Patienten oder bei Vorliegen einer syndromalen Adipositas müssen die ethischen Aspekte, eventuell unter Hinzuziehen der Ethikkommission, diskutiert werden.

\section{Operationstechniken}

Bei einer systematischen Analyse der Literatur in der Datenbank PubMed (1994-2007) zur Adipositaschirurgie bei Kindern und Jugendlichen fanden sich 802 Literaturstellen, davon 47 relevante Artikel (3 Kohortenstudien, 15 Fallserien, 6 Fallbeschreibungen, 13 Übersichtsarbeiten, 4 Leitlinien und 6 Leserbriefe bzw. Expertenmeinungen). Betrachtet man 22 ausgewählte Originalarbeiten detaillierter, so waren die mit Abstand häufigsten Operationsverfahren das «Banding» des Magens (kumulativ $\mathrm{n}=274$, davon 248 laparoskopisch) und der Roux-Y-Magenbypass (kumulativ $\mathrm{n}=292$, davon 62 laparoskopisch). Technische Details, wie Trokarpositionen, Positionierungen des Bandes und Größe des Pouches beim Bypass, entsprachen im Wesentlichen den Berichten aus der Erwachsenenliteratur. Die häufigsten Komplikationen beim Magenband waren Dislokation $(1,8 \%)$, Notwendigkeit der Entfernung (aus vielfältigen Gründen, 2,1\%), Eisenmangelanämie (1,5\%) und Haarausfall (1,8\%). Beim Roux-Y-Bypass fanden sich Ulzera und Bauchwandhernien (je 2,4\%), Eisenmangel (3,4\%) und Proteinmangel (4,8\%). Revisionen waren in 7,5\% der Fälle notwendig. Alle Verfahren waren im Hinblick auf die postoperative Gewichtsabnahme ähnlich effektiv und ermöglichten einen stabilen Gewichtsverlust über mehrere Jahre hinweg. 
Als aktuelle Entwicklung konnte im Jahr 2008 erstmals über die erfolgreiche Schlauchmagenbildung (sleeve gastrectomy) als «Standalone-Technik» [13] bei einer kleinen Serie von Kindern und Jugendlichen (mittleres Alter 14,5 Jahre (Range 8-17), mittlerer präoperativer BMI 48,4 kg/m² (Range 40,6-56,3), mehrere Begleiterkrankungen im Sinne des metabolischen Syndroms) bericht werden. Dabei wurde auf Empfehlung der Experten [14, 15] besonders darauf geachtet, dass der Restpouch maximal ein Volumen von circa $100 \mathrm{ml}$ hat. Die kurzfristigen Ergebnisse (Range 6-19 Monate) waren ermutigend: Es traten keine postoperativen Probleme auf, alle Patienten hatten signifikant an Gewicht verloren (mittlerer BMI dann bei $37,2 \mathrm{~kg} / \mathrm{m}^{2}$ ), und es erfolgte eine stetige Besserung bis hin zur vollständigen Rückbildung einiger Begleiterkrankungen. Bemerkenswert war ferner, dass innerhalb des ersten postoperativen Jahres keine Zeichen einer Mangelernährung oder eines Vitaminmangels gefunden wurden, wobei alle Patienten postoperativ nach erfolgtem Kostaufbau eine Substitution erhalten.

\section{Diskussion}

Die bariatrische Chirurgie stellt auch in Deutschland eine sinnvolle Therapieoption für ausgewählte Kinder und Jugendliche mit morbider Adipositas dar. Allerdings sollte deren Behandlung in
Anlehnung an amerikanische Modelle (z. B. Cincinnati Children's) in ausgewiesenen, möglichst pädiatrischen Zentren erfolgen, die zunächst alle konservativen Maßnahmen ausschöpfen und nur die frustranen Fälle zur Diskussion stellen. Die Indikationsstellung für einen Eingriff ist international etabliert und sollte nun auch für Deutschland in Form einer Leitlinie zügig definiert werden, da sowohl der Roux-Y-Bypass als auch das Magenband und neuerdings die Sleeve Gastrectomy zu einer effektiven postoperativen Gewichtsabnahme und Besserung der komorbiden Erkrankungen zu führen scheinen. Allerdings bleiben die Erfahrungen und insbesondere die langfristigen Komplikationen der einzelnen Operationsmethoden im Jugendalter begrenzt [16-20]. Deswegen wäre es sinnvoll, dass die Adipositaschirurgie bei Kindern und Jugendlichen nicht vereinzelt und neben den Erwachsenen, sondern in ausgewiesenen Zentren stattfindet, die Patienten jeden Lebensalters behandeln und kontinuierlich nachbetreuen. Damit könnten Daten zu der Sicherheit, den Vor- und Nachteilen und den Komplikationen und Langzeitergebnissen ermittelt und die aktuellen Konzepte zur morbiden Adipositaschirurgie bei Kindern und Jugendlichen ständig kontrolliert und verbessert werden [16].

\section{Disclosure}

Die Autoren geben keine Interessenkonflikte an.

\section{Literatur}

1 Sinha R, Fisch G, Teague B, Tamborlane WV, Banyas B, Allen K, Savoye M, Rieger V, Taksali S, Barbetta G, Sherwin RS, Caprio S: Prevalence of impaired glucose tolerance among children and adolescents with marked obesity. N Engl J Med 2002;346(11):802-810.

- 2 Kalies H, Lenz J, von Kries R: Prevalence of overweight and obesity and trends in body mass index in German pre-school children, 1982-1997. Int J Obes Relat Metab Disord 2002;26(9):12111217.

3 Kiess W, Bottner A, Blüher S, Raile K, Seidel B Kapellen TM, Keller E, Kratzsch J: Pharmacoeconomics of obesity management in childhood and adolescence. Expert Opin Pharmacother 2003;4(9):1471-1477.

4 Reich A, Müller G, Gelbrich G, Deutscher K Gödicke R, Kiess W: Obesity and blood pressure - results from the examination of 2365 schoolchildren in Germany. Int J Obes Relat Metab Disord 2003;27(12):1459-1464.

5 Kiess W, Böttner A, Blüher S, Raile K, Galler A, Kapellen TM: Type 2 diabetes mellitus in children and adolescents - the beginning of a catastrophe? Nephrol Dial Transplant 2004;19(11):26932696.

6 Blüher S, Kiess W: Obesity and type 2 diabetes mellitus in childhood and adolescence; in Mantzoros C (ed): Obesity and Diabetes. Totowa, NJ, Humana Press, 2005.

7 Kromeyer-Hauschild K, Zellner K: Trends in overweight and obesity and changes in the distribution of body mass index in schoolchildren of Jena, East Germany. Eur J Clin Nutr 2007;61(3) 404-411.
Maggard MA, Shugarman LR, Suttorp M, Maglione M, Sugerman HJ, Livingston EH, Nguyen NT, Li Z, Mojica WA, Hilton L, Rhodes S, Morton SC, Shekelle PG: Meta-analysis: surgical treatment of obesity. Ann Intern Med 2005; 142(7):547-559.

-9 Sjöström L, Narbro K, Sjöström CD, Karason K, Larsson B, Wedel H, Lystig T, Sullivan M, Bouchard C, Carlsson B, Bengtsson C, Dahlgren S, Gummesson A, Jacobson P, Karlsson J, Lindroos AK, Lönroth H, Näslund I, Olbers T, Stenlöf K, Torgerson J, Ågren, Carlsson LMS: Effects of bariatric surgery on mortality in Swedish obese subjects. N Engl J Med 2007;357:741-752.

10 Adams TD, Gress RE, Smith SC, Halverson RC, Simper SC, Rosamond WD, LaMonte MJ, Stroup AM, Hunt SC: Long-term mortality after gastric bypass surgery. N Engl J Med 2007;357:753-761.

11 Inge TH, Krebs NF, Garcia VF, Skelton JA, Guice KS, Strauss RS, Albanese CT, Brandt ML, Hammer LD, Harmon CM, Kane TD, Klish WJ, Oldham KT, Rudolph CD, Helmrath MA, Donovan E, Daniels SR: Bariatric surgery for severely overweight adolescents: concerns and recommendations. Pediatrics 2004;114:217-223.

12 Fried M, Hainer V, Basdevant A, Buchwald H, Deitel M, Finer N, Greve JW, Horber F, MathusVliegen E, Scopinaro N, Steffen R, Tsigos C, Weiner R, Widhalm K: Interdisciplinary European guidelines for surgery for severe (morbid) obesity. Obes Surg 2007;17(2):260-270.

13 Till H, Blüher S, Hirsch W, Kiess W: Efficacy of laparoscopic sleeve gastrectomy (LSG) as a stand-alone technique for children with morbid obesity. Obes Surg 2008;18(8):1047-1049.
14 Weiner RA, Weigand G, Weiner S, Theodoridou S, Pomhoff I: Laparoskopische Schlauchmagenbildung in der Behandlung der morbiden Adipositas. Adipositas 2008;2(1):11-18.

15 Deitel M, Crosby RD, Gagner M: The First International Consensus Summit for Sleeve Gastrectomy (SG), New York City, October 25-27, 2007. Obes Surg 2008;18(5):487-496.

16 Helmrath MA, Brandt ML, Inge TH: Adolescent obesity and bariatric surgery. Surg Clin North Am 2006;86(2):441-454.

17 Inge TH, Xanthakos SA, Zeller MH: Bariatric surgery for pediatric extreme obesity: now or later? Int J Obes 2007;31:1-14.

18 Stanford A, Glascock JM, Eid GM, Kane T, Ford HR, Ikramuddin S, Schauer P: Laparoscopic Roux-en-Y gastric bypass in morbidly obese adolescents. J Pediatr Surg 2003;38(3):430-433.

19 Sugerman HJ, Sugerman EL, DeMaria EJ, Kellum JM, Kennedy C, Mowery Y, Wolfe LG: Bariatric surgery for severely obese adolescents. J Gastrointest Surg 2003;7(1):102-108.

20 Dolan K, Creighton L, Hopkins G, Fielding G: Laparoscopic gastric banding in morbidly obese adolescents. Obes Surg 2003;13(1):101-104.

21 Blüher S, Münsterer O, Beckert-Zieglschmid C, Rohde C, Blüher M, Stumvoll M, von Klitzing K, Till H, Kiess W: Adipositas-Chirurgie bei morbid adipösen Jugendlichen: Vorschlag für ein interdisziplinäres Konzept. Adipositas 2008;2(1):3640 . 